\title{
Calculating limits and colimits in pro-categories
}

\author{
by \\ Daniel C. Isaksen (Notre Dame, IN)
}

\begin{abstract}
We present some constructions of limits and colimits in pro-categories. These are critical tools in several applications. In particular, certain technical arguments concerning strict pro-maps are essential for a theorem about étale homotopy types. We also correct some mistakes in the literature on this topic.
\end{abstract}

1. Introduction. Pro-categories have found various uses over the past few decades, from algebraic geometry [SGA4], [AM], [F] to shape theory $[\mathrm{CP}],[\mathrm{EH}],[\mathrm{MS}]$ to geometric topology [CJS] to applied mathematics [CP, Appendix]. Unfortunately, the definition of a pro-category is subtle and complicated. The technical complexity of many papers using pro-categories bears this out. As an example of the subtlety of pro-categories, it was only recently discovered [I1] that the category pro- $\mathcal{C}$ is cocomplete whenever $\mathcal{C}$ is cocomplete. (Despite some claims in the literature, [AM, App. 4.3] shows that pro- $\mathcal{C}$ is cocomplete only when $\mathcal{C}$ is small, which is useful for categories like pro-finite groups but not useful for categories like pro-sets or pro-spaces.)

In several recent projects involving pro-categories [CI], [I1]-[I3], we have found it necessary to compute various limits and colimits. Since these different computations are similar, we have collected them together in this article. We hope that these calculational tools will be useful to others studying pro-categories.

We begin in Section 2 with a review of the necessary background on pro-categories. We emphasize two important and well known facts about pro-objects because they are central to the techniques in this paper. First,

2000 Mathematics Subject Classification: 18A30, 18Exx, 55U35, 14F35.

Key words and phrases: pro-category, limit, colimit, essentially of type $C$, pro-abelian category.

The author was supported by an NSF Postdoctoral Fellowship. The author thanks Dan Christensen for his constructive comments, especially concerning Remark 4.2 and Section 7. 
every pro-object is isomorphic to a pro-object indexed by a cofinite directed set. Second, every map in a pro-category has a level representation.

The category of arrows $\operatorname{Ar}$ (pro- $\mathcal{C}$ ) in a pro-category is equivalent to the pro-category pro-(Ar $\mathcal{C})[\mathrm{Me}]$. More generally, for any finite loopless category $A$, the functor category (pro- $\mathrm{C})^{A}$ is equivalent to pro- $\left(\mathrm{C}^{A}\right)$. We introduce in Section 3 a new reindexing result for infinite diagrams that are cofinite (see Definition 2.2). The categories (pro- $\mathrm{C})^{A}$ and pro- $\left(\mathcal{C}^{A}\right)$ are not equivalent, but there is an essentially surjective functor

$$
\text { pro- }\left(\mathrm{C}^{A}\right) \rightarrow(\text { pro-e })^{A} \text {. }
$$

Fortunately this suffices for our purposes; it means that every diagram in pro- $C$ has a level representation.

When $\mathcal{C}$ is small and $A$ has finite morphism sets, the functor of the previous paragraph is again essentially surjective. Morel $[\mathrm{Mo}]$ assumed that the functor in the previous paragraph gives an equivalence between the categories of pro-simplicial finite sets and simplicial pro-finite sets. In fact, this is not true, as shown in Example 3.7.

In Section 3.1, we specialize to a particular situation involving diagrams of strict pro-maps. This is critical for a theorem about hypercover descent for the étale topological type [I2].

With this reindexing result in hand, we give in Section 4 an explicit description of cofiltered limits in pro-categories. Since every limit can be rewritten in terms of finite limits of loopless diagrams and cofiltered limits, one can in principle describe an arbitrary limit if one can describe these two special kinds.

Calculation of finite limits of loopless diagrams in pro-categories is well known [AM, App. 4.2]. One finds a level representation for the diagram and then takes the levelwise limit. Calculation of cofiltered limits in procategories is more complicated. The description of these limits in Theorem 4.1 is the essential result of this paper.

This leads to the main applications. Given a class of maps $C$, a pro-map is essentially of type $C$ if it has a level representation by maps in $C$. This kind of pro-map plays a significant role in many studies involving pro-categories. For example, it is central to the abelian structure on the category pro- $\mathcal{A}$ for any abelian category $\mathcal{A}[\mathrm{AM}],[\mathrm{D}],[\mathrm{P}],[\mathrm{V}]$. Also, it is an important part of various closed model structures on pro-categories [CI], [EH], [I1], [I3].

We prove in Section 5 that for any class $C$, filtered limits and retracts preserve the maps that are essentially of type $C$. This fact has several immediate corollaries, which we explore in Section 6. The most interesting is that filtered limits commute with finite colimits in any pro-category. In other words, filtered limits are exact. This is opposite to the behavior of most familiar categories. Consequently, filtered limits are exact in the abelian 
category pro- $\mathcal{A}$ when $\mathcal{A}$ is any abelian category. This fact was observed in [AHJM, §2] without proof. Thus, pro- $\mathcal{A}$ is very different from familiar abelian categories such as abelian groups, $R$-modules, presheaves, or sheaves. The category pro- $\mathcal{A}$ has many of the properties of the opposites of these familiar abelian categories, even though it is not equivalent to any of them. Example 6.4 shows that filtered colimits are not exact for pro-abelian groups.

Another application concerns cocompactness. Most familiar categories do not have any cocompact objects, but we show in Section 7 that pro-categories have lots of them. This is an important technical ingredient for [CI].

In the last section, we make some constructions of colimits in pro-categories. In general, such colimits are very messy. The constructions here are about as simple and concrete as possible.

We make one brief comment about ind-categories. All the results of this paper dualize because ind- $\mathcal{C}$ is equivalent to (pro- $\left.{ }^{\mathrm{ep}}\right)^{\mathrm{op}}$. For example, we could describe filtered colimits in ind-categories. We give no details because ind-categories occur less often in our intended applications. See [CI] for one exception.

\section{Preliminaries on pro-categories}

Definition 2.1. For a category $\mathcal{C}$, the category pro- $\mathcal{C}$ has objects all cofiltering diagrams in $\mathcal{C}$, and

$$
\operatorname{Hom}_{\text {pro-e }}(X, Y)=\lim _{s} \operatorname{colim}_{t} \operatorname{Hom}_{\mathcal{C}}\left(X_{t}, Y_{s}\right) .
$$

Composition is defined in the natural way.

A category $I$ is cofiltering if the following conditions hold: it is non-empty and small; for every pair of objects $s$ and $t$ in $I$, there exists an object $u$ together with maps $u \rightarrow s$ and $u \rightarrow t$; and for every pair of morphisms $f$ and $g$ with the same source and target, there exists a morphism $h$ such that $f h$ equals $g h$. Recall that a category is small if it has only a set of morphisms. A diagram is said to be cofiltering if its indexing category is so.

Objects of pro- $\mathcal{C}$ are functors from cofiltering categories to $\mathcal{C}$. We use both set theoretic and categorical language to discuss indexing categories; hence " $t \geq s$ " and " $t \rightarrow s$ " mean the same thing when the indexing category is actually a directed set.

The word "pro-object" refers to objects of pro-categories. A constant pro-object is one indexed by the category with one object and one (identity) map. Let $c: \mathcal{C} \rightarrow$ pro- $\mathcal{C}$ be the functor taking an object $X$ to the constant pro-object with value $X$. Note that this functor makes $\mathcal{C}$ a full subcategory of pro-e. The limit functor $\lim ^{\mathrm{e}}:$ pro- $\mathrm{C} \rightarrow \mathcal{C}$ is the right adjoint of $c$. We shall always write this functor as $\lim ^{\mathrm{e}}$ to distinguish it from the functor $\lim ^{\text {pro }}$, which is the limit internal to the category pro-e. 
Let $Y: I \rightarrow \mathcal{C}$ and $X: J \rightarrow \mathcal{C}$ be arbitrary pro-objects. We say that $X$ is cofinal in $Y$ if there is a cofinal functor $F: J \rightarrow I$ such that $X$ is equal to the composite $Y F$. This means that for every $s$ in $I$, the overcategory $F \downarrow s$ is cofiltered. In the case when $F$ is an inclusion of directed sets, $F$ is cofinal if and only if for every $s$ in $I$ there exists a $t$ in $J$ such that $t \geq s$. The importance of this definition is that $X$ is isomorphic to $Y$ in pro- $\mathrm{C}$.

A level representation of a map $f: X \rightarrow Y$ is: a cofiltered index category $I$; pro-objects $\widetilde{X}$ and $\widetilde{Y}$ indexed by $I$ and isomorphisms $X \rightarrow \widetilde{X}$ and $Y \rightarrow \widetilde{Y}$; and a collection of maps $f_{s}: \widetilde{X}_{s} \rightarrow \widetilde{Y}_{s}$ for all $s$ in $I$ such that for all $t \rightarrow s$ in $I$, there is a commutative diagram

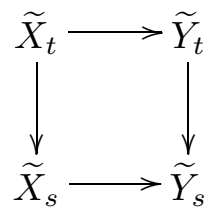

and such that the maps $f_{s}$ represent a pro-map $\tilde{f}: \widetilde{X} \rightarrow \widetilde{Y}$ belonging to a commutative square

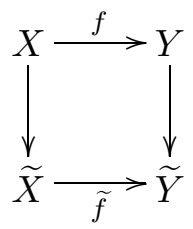

in pro-C. That is, a level representation is just a natural transformation such that the maps $f_{s}$ represent the element $f$ of

$$
\lim _{s} \operatorname{colim}_{t} \operatorname{Hom}_{\mathcal{C}}\left(X_{t}, Y_{s}\right) \cong \lim _{s} \operatorname{colim}_{t} \operatorname{Hom}_{\mathcal{C}}\left(\widetilde{X}_{t}, \widetilde{Y}_{s}\right) .
$$

Every map has a level representation [AM, Appendix 3.2], [Me].

More generally, suppose given any diagram $A \rightarrow$ pro- $\mathrm{C}: a \mapsto X^{a}$. A level representation of $X$ is: a cofiltered index category $I$; a functor $\widetilde{X}: A \times I \rightarrow$ $\mathcal{C}:(a, s) \mapsto \widetilde{X}_{s}^{a}$; and isomorphisms $X^{a} \rightarrow \widetilde{X}^{a}$ such that for every map $\phi: a \rightarrow b$ in $A, \widetilde{X}^{\phi}$ is a level representation for $X^{\phi}$. In other words, $\widetilde{X}$ is a uniform level representation for all the maps in the diagram $X$. Not every diagram of pro-objects has a level representation.

Suppose that $X: I \rightarrow \mathcal{C}$ and $Y: J \rightarrow \mathcal{C}$ are two pro-objects. A strict representation [F, p. 36] of a map $f: X \rightarrow Y$ is a functor $F: J \rightarrow I$ and a natural transformation $\eta: X \circ F \rightarrow Y$ such that the maps $\eta_{s}: X_{F(s)} \rightarrow Y_{s}$ represent the element $f$ of $\lim _{s} \operatorname{colim}_{t} \operatorname{Hom}_{\mathcal{C}}\left(X_{t}, Y_{s}\right)$.

More generally, a strict representation of a diagram $X$ in pro- $\mathrm{C}$ consists of strict representations $\left(F^{\phi}, \eta^{\phi}\right)$ for every map $\phi$ in $X$ such that for every pair of composable maps $\phi$ and $\psi$ in $X$, the functor $F^{\psi \phi}$ equals $F^{\phi} F^{\psi}$ and the natural transformation $\eta^{\psi \phi}$ equals $\eta^{\psi} \circ \eta^{\phi} F^{\psi}$. 
If $C$ is a class of objects, then a pro-object $X$ is of type $C$ if each $X_{s}$ belongs to $C$. A pro-object is essentially of type $C$ if it is isomorphic to a pro-object of type $C$. Similarly, a level representation $\widetilde{X} \rightarrow \widetilde{Y}$ of a map $X \rightarrow Y$ is of type $C$ (where $C$ is a class of maps) if each $\widetilde{X}_{s} \rightarrow \widetilde{Y}_{s}$ belongs to $C$. A map is essentially of type $C$ if it has a level representation of type $C$.

Definition 2.2. A category $A$ is loopless if it has no non-identity endomorphisms. A category $A$ is cofinite if it is small, loopless, and for every object $a$ of $A$, only finitely many arrows have source $a$.

A pro-object or level representation is cofinite directed if it is indexed by a cofinite directed set.

For every cofiltered category $J$, there exists a cofinite directed set $I$ and a cofinal functor $I \rightarrow J$ [AR, Th. 1.5]. Therefore, every pro-object is isomorphic to a cofinite directed pro-object. Similarly, every map has a cofinite directed level representation. Thus, it is possible to restrict the definition of a pro-object to only consider cofinite directed sets as index categories. However, we find this unnatural. Many general notions of procategories are best expressed in terms of cofiltered categories, not in terms of cofinite directed sets. For example, consider $[\mathrm{P}]$, in which the author assumes that all pro-objects are indexed by directed sets. The construction of limits on pages $12-13$ is technically correct, but it produces a pro-object that is not indexed by a directed set!

On the other hand, we find it much easier to work with cofinite directed pro-objects in practice. Thus, most of our results start by assuming without loss of generality that a pro-object is indexed by a cofinite directed set. Cofiniteness is critical because many arguments and constructions proceed inductively.

3. Level representations. We study in this section the question of replacing diagrams of pro-objects with level representations. We use level representations in Section 4 to construct cofiltered limits.

One of the fundamental tools for studying pro-categories is the fact that every morphism in a pro-category has a level representation. In fact, every finite loopless diagram in a pro-category has a level representation [AM, App. 3.3]. The following result [Me] is an elegant explanation of these level representation principles.

TheOREm 3.1. Let $A$ be any finite loopless category, and let $\mathcal{C}$ be any category. There is a full, faithful, and essentially surjective functor pro- $\left(\mathrm{C}^{A}\right)$ $\rightarrow(\text { pro- } \mathrm{C})^{A}$.

Recall that a functor is essentially surjective if every object in the target is isomorphic to an object in the image of the functor. 
When $A$ is the category with two objects and one non-identity morphism, the theorem just says that every pro-map has a level representation. This special case will be useful below in Corollaries 5.2 and 5.6.

Beware that the pro-objects of $[\mathrm{Me}]$ are indexed by cofiltered categories that are not necessarily small. On the other hand, all of our indexing categories are small; this is an important distinction to keep in mind. Nevertheless, Theorem 3.1 (and its consequences) are still true.

One important application of Theorem 3.1 is the construction of finite limits in pro- $\mathrm{C}$ as stated in the next proposition from [AM, App. 4.2].

Proposition 3.2. Let $\mathcal{C}$ be a category with finite limits. Let $A$ be a finite loopless category, and let $X: A \rightarrow$ pro- $\mathrm{C}$ be a diagram in pro-C. Let $\widetilde{X}: A \times I \rightarrow \mathcal{C}$ be a level representation, where $I$ is some cofiltered category, and let $Z$ be the pro-object indexed by $I$ given by $Z_{s}=\lim _{a}^{\mathrm{e}} \widetilde{X}_{s}^{a}$. Then $Z$ is isomorphic to $\lim _{a}^{\mathrm{pro}} X^{a}$.

From this point, one can generalize in different directions. For us the most interesting situation occurs when $A$ is a cofinite category as described in Definition 2.2. Every finite loopless category is cofinite, but other infinite categories may also satisfy these conditions. The most important examples for our purposes are cofinite directed sets. We give more examples of cofinite categories in Section 8 when we study colimits of pro-objects.

Objects of pro- $\left(\mathfrak{C}^{A}\right)$ are level representations of diagrams in pro- $\mathcal{C}$. This viewpoint gives us a functor $F:$ pro- $\left(\mathcal{C}^{A}\right) \rightarrow(\text { pro- } \mathcal{C})^{A}$.

TheOREm 3.3. If $A$ is a cofinite category, then the functor $F$ is essentially surjective.

Proof. Let $X: A \rightarrow$ pro-C : $a \mapsto X^{a}$ be a diagram of pro-objects. We want to show that the diagram $X$ has a level representation. We may assume that each pro-object $X^{a}$ is cofinite directed with index set $I^{a}$. Choose $I$ to be an arbitrary cofinite directed set with cardinality greater than or equal to the cardinalities of every $I^{a}$; this will be the index set for the level representation $\widetilde{X}$. Also choose arbitrary set surjections $h^{a}: I \rightarrow I^{a}$.

For every $a$, we build a new pro-object $\widetilde{X}^{a}$ by constructing a cofinal function $f^{a}: I \rightarrow I^{a}$ and letting $\widetilde{X}_{s}^{a}$ equal $X_{f^{a}(s)}^{a}$.

Fix an element $a$ of $A$. Assume that the function $f^{b}$ has already been constructed on all indices $b$ for which there exists a map $a \rightarrow b$ in $A$. Then we may proceed by induction because $A$ is cofinite.

We may define $f^{a}$ inductively since $I$ is cofinite. Let $s$ be an index in $I$, and suppose that $f^{a}$ has already been defined for $t<s$. We choose $f^{a}(s)$ satisfying the following properties. This is possible because there are only finitely many conditions. 
First, choose $f^{a}(s)$ sufficiently large so that $f^{a}(t) \leq f^{a}(s)$ for all $t<s$. This guarantees that $\widetilde{X}^{a}$ is a pro-object.

Second, choose $f^{a}(s)$ large enough so that $f^{a}(s) \geq h^{a}(s)$. This guarantees that $f^{a}$ is cofinal so that the natural map $X^{a} \rightarrow \widetilde{X}^{a}$ is an isomorphism.

Third, choose $f^{a}(s)$ large enough so that for all maps $a \rightarrow b$ in $A$, there are maps $X_{f^{a}(s)}^{a} \rightarrow X_{f^{b}(s)}^{b}$ representing $X^{a} \rightarrow X^{b}$ such that the diagrams

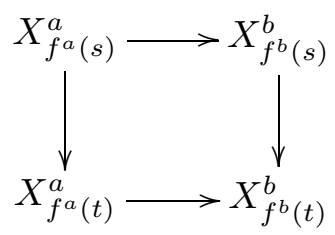

commute for all $t<s$. This guarantees that $\widetilde{X}^{a} \rightarrow \widetilde{X}^{b}$ is a level representation.

Finally, choose $f^{a}(s)$ large enough so that for all pairs of arrows $a \rightarrow b$ and $b \rightarrow c$ in $A$, the diagram

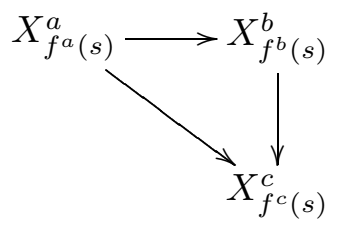

commutes. This guarantees that $\widetilde{X}$ is a level representation.

Note that the isomorphisms $X^{a} \rightarrow \widetilde{X}^{a}$ are given by representatives

$$
X_{f^{a}(s)}^{a} \rightarrow X_{f^{a}(s)}^{a}=\widetilde{X}_{s}^{a}
$$

that are identity maps. It follows that the diagram

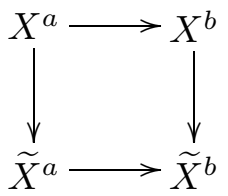

commutes for every map $a \rightarrow b$ in $A$ because both compositions are given by representatives

$$
X_{f^{a}(s)}^{a} \rightarrow X_{f^{b}(s)}^{b}=\widetilde{X}_{s}^{b} .
$$

REMARK 3.4. In general, the functor $F$ is not full and faithful unless $A$ is finite. We briefly explain why. Let $X$ and $Y$ be any two objects of pro- $\left(\mathrm{C}^{A}\right)$. Then

$$
\operatorname{Hom}(X, Y)=\lim _{s} \operatorname{colim}_{t} \operatorname{End}_{a} \operatorname{Hom}\left(X_{t}^{a}, Y_{s}^{a}\right)
$$


where End refers to the usual end construction for calculating morphisms in a diagram category [ML, $\S \mathrm{IX} .5]$. On the other hand,

$$
\operatorname{Hom}(F X, F Y)=\operatorname{End}_{a} \lim _{s} \operatorname{colim} \operatorname{Hom}\left(X_{t}^{a}, Y_{s}^{a}\right) \text {. }
$$

The limit involved in calculating the end does not commute with the colimit unless it is a finite limit. The end is a finite limit if and only if $A$ is a finite category.

Theorem 3.3 gives us level representations for arbitrary cofinite indexing categories. Under other hypotheses, one can make stronger statements. We explore some of these situations below.

Assume that $\mathcal{C}$ is a small category that has finite limits. We define another functor

$$
G:(\text { pro-e })^{A} \rightarrow \text { pro- }\left(\mathrm{C}^{A}\right)
$$

as follows. Let $X$ be an object of $(\text { pro- } \mathcal{C})^{A}$. Let $I$ be the category of all morphisms in (pro- $\mathrm{C})^{A}$ of the form $X \rightarrow c Y$, where $Y$ varies over all objects of $\mathrm{e}^{A}$. Note that $\mathrm{e}^{A}$ has only a set of objects because $\mathcal{C}$ and $A$ are both small. We are abusing notation here; strictly speaking $c Y$ is an object of pro- $\left(\mathrm{C}^{A}\right)$, but we are identifying it with its image in (pro- $\left.\mathrm{C}\right)^{A}$ under the functor $F$ defined before Theorem 3.3.

Since $\mathcal{C}$ has finite limits, so does $\mathcal{C}^{A}$. This means that $I$ is cofiltered. There is a functor $I \rightarrow \mathrm{e}^{A}$ taking a morphism $X \rightarrow c Y$ to $Y$. This is the object $G X$ of pro- $\left(\mathrm{e}^{A}\right)$.

In constructing $G$, it is very important that $\mathcal{C}$ be small. Otherwise, the cofiltered category $I$ is not small.

LEMma 3.5. If $\mathcal{C}$ is small and has finite limits, then $G$ is the left adjoint of $F$.

Proof. The object $G X$ was constructed to have the correct universal property.

The next proposition tells us that $F$ is essentially surjective, so every diagram in (pro-e $)^{A}$ has a level representation.

Proposition 3.6. Let $\mathcal{C}$ be a small category with finite limits and let $A$ be a small indexing category whose morphism sets are finite. Then the composition FG is naturally isomorphic to the identity on (pro-e $)^{A}$.

Proof. By adjointness, there is a map $X \rightarrow F G X$. It suffices to show that for each $a$, the map $X^{a} \rightarrow(F G X)^{a}$ is an isomorphism in pro-e. We will explicitly construct an inverse.

The pro-object $(F G X)^{a}$ can be described as follows. Its index set $I$ is the collection of all morphisms $X \rightarrow c Y$ in (pro- $\mathrm{C})^{A}$, where $Y$ varies over all 
objects of $\mathcal{C}^{A}$. The pro-object $(F G X)^{a}: I \rightarrow \mathcal{C}$ takes the element $X \rightarrow c Y$ of $I$ to $Y^{a}$.

In order to construct the inverse $(F G X)^{a} \rightarrow X^{a}$, we must give for each $t$ a diagram $Y_{t}$ in $\mathcal{C}^{A}$, a morphism $X \rightarrow c Y_{t}$ in $(\text { pro- } \mathcal{C})^{A}$, and a map $Y_{t}^{a} \rightarrow X_{t}^{a}$.

Let $Y_{t}$ be the cofree diagram with value $X_{t}^{a}$ at $a$. This means that $Y_{t}^{b}$ equals $\prod_{a \rightarrow b} X_{t}^{a}$, where the product ranges over all morphisms from $a$ to $b$ in $A$. These products exist because $\mathcal{C}$ has finite limits and because $A$ has finite morphism sets. Now there is a canonical map $X \rightarrow c Y_{t}$ induced by the map $X^{a} \rightarrow c X_{t}^{a}$. Finally the map $Y_{t}^{a} \rightarrow X_{t}^{a}$ is given by projection onto the factor corresponding to the identity map $a \rightarrow a$.

EXAMPLE 3.7. The standard simplicial indexing category $\Delta$ has finite morphism sets, so the above proposition applies. We conclude that the category of simplicial pro-finite sets is a retract of the category of pro-(simplicial finite sets), but these categories are not equivalent.

We give a specific example showing that $F$ is not faithful. For each $n \geq 0$, let $S^{n}$ be the simplicial finite set $\Delta[n] / \partial \Delta[n]$. For $0 \leq k \leq \infty$, let $T_{k}$ be the wedge $\bigvee_{n=0}^{k} S^{n}$. Even when $k=\infty$, the simplicial set $T_{k}$ is finite in each dimension. Now let $X$ be the pro-simplicial finite set $c T_{\infty}$, and let $Y$ be the pro-simplicial finite set

$$
\ldots \rightarrow T_{k+1} \rightarrow T_{k} \rightarrow \ldots \rightarrow T_{1} \rightarrow T_{0},
$$

where the structure maps consist of collapsing a sphere to the basepoint. It is easy to check that $X$ and $Y$ are not isomorphic as pro-simplicial finite sets, but $F X$ and $F Y$ are both equal to $T_{\infty}$ considered as a simplicial pro-finite set.

The distinction between simplicial pro-finite sets and pro-simplicial finite sets is blurred in $[\mathrm{Mo}]$, so the reader must interpret statements there very carefully to avoid confusion. Proposition 3.6 tells us $F$ is essentially surjective, so every simplicial pro-finite set can be represented by a pro-simplicial finite set.

The trouble described in the previous example arises because the indexing category $\Delta$ has infinitely many objects. The following proposition makes this clear.

Proposition 3.8. Let $\mathcal{C}$ be a small category that has finite limits, and let $A$ be an arbitrary finite indexing category. Then the functor

$$
F: \text { pro- }\left(\mathcal{C}^{A}\right) \rightarrow(\text { pro- } \mathcal{C})^{A}
$$

is an equivalence of categories.

Proof. The proof is essentially the same as [Me, $\S 4]$, but beware that our pro-objects are always indexed by small categories. 
EXAMPLE 3.9. Let $G$ be a finite group. Then the above proposition tells us that the category of $G$-pro-finite sets is equivalent to the category of pro- $G$-finite sets. Similarly, the category of $G$-pro-simplicial finite sets is equivalent to the category of pro- $G$-simplicial finite sets.

3.1. Level representations for strict diagrams. The reindexing of Theorem 3.3 is very general but also not canonical; one must make choices everywhere. We next study a specific situation in which the reindexing is more natural. This situation arises in [I2].

Suppose given a strict representation of a diagram $X$ indexed by $A$. Also suppose that $A$ is cofinite and that the index category $I^{a}$ of each $X^{a}$ has finite limits. If $I^{a}$ is a directed set, then this means that every pair of elements in $I^{a}$ has a least upper bound. For all arrows $\phi: a \rightarrow b$ in $A$, we have functors $I^{\phi}: I^{a} \rightarrow I^{b}$ between index categories.

We now discuss a natural way of constructing the reindexing of Theorem 3.3. Let $I$ be the product of the categories $I^{a}$ for which $a$ is the source of no non-identity maps. The idea is that $I$ is the product of the indexing categories of all objects "at the bottom" of the diagram. Note that $I$ is cofiltered since arbitrary products preserve cofiltered categories.

For every $s=\left(s^{a}\right)$ in $I$, define $\widetilde{X}_{s}^{a}$ to be $X_{t}^{a}$, where

$$
t=\lim _{\phi: a \rightarrow b} I^{\phi}\left(s^{b}\right) .
$$

The limit is calculated in the category $I^{a}$. The idea is that $t$ is the smallest common refinement of the elements $I^{\phi}\left(s^{b}\right)$ in $I^{a}$. It is straightforward to check that this definition satisfies all of the conditions of Theorem 3.3.

EXAMPLE 3.10. The main motivation for this discussion of strict representations is that it is critical for the proof of the hypercover descent theorem for étale topological types [I2].

For every scheme $X$, let $\operatorname{HRR}(X)$ be the category of rigid hypercovers of $X$ [F, Prop. 4.3], [I2]. This is the cofiltered index category for the étale topological type Et $X$ of $X$, which is a pro-space. Rigid limits [I2] give us finite limits in $\operatorname{HRR}(X)$.

For every scheme map $f: X \rightarrow Y$, rigid pullback [I2] induces a functor $f^{*}: \operatorname{HRR}(Y) \rightarrow \operatorname{HRR}(X)$. For every rigid hypercover $U$ of $Y$, there is a canonical rigid hypercover map $f^{*} U \rightarrow U$. This induces a strict representation of the map Et $X \rightarrow$ Et $Y$ of pro-spaces.

The rigid pullback functors are compatible under composition in the sense that $(g f)^{*}=f^{*} g^{*}$. Therefore, every diagram of schemes induces a strict representation of a diagram of pro-spaces. Hence, we can apply the ideas of this discussion to reindexing the étale topological types of cofinite diagrams of schemes. 
4. Cofiltered limits. We can now give a model for constructing cofiltered limits in pro-categories. Start with a functor $X: B \rightarrow$ pro- $\mathrm{C}$, where $B$ is a cofiltered index category. As mentioned in Section 2, there exists a cofinite directed set $A$ and a cofinal functor $A \rightarrow B$. We may replace $X$ with the composition $A \rightarrow B \rightarrow$ pro- $\mathcal{C}$ because the limit does not change. Since $A$ is a cofinite category, we can construct a level representation $\widetilde{X}$ of $X$ as given in Theorem 3.3. A product of cofiltered categories is again a cofiltered category, so the functor $\widetilde{X}: A \times I \rightarrow \mathcal{C}$ can be viewed as a pro-object.

TheOREM 4.1. The pro-object $\widetilde{X}$ is isomorphic to $\lim _{a}^{\text {pro }} X^{a}$.

Proof. Because each $\widetilde{X}^{a}$ is isomorphic to $X^{a}$, it suffices to show that $\widetilde{X}$ is isomorphic to $\lim _{a}^{\text {pro }} \widetilde{X}^{a}$. By direct calculation with the definition of morphism sets for pro- $\mathcal{C}, \widetilde{X}$ satisfies the required universal property.

REMARK 4.2. The reindexing provided by Theorem 3.3 is not absolutely necessary to construct cofiltered limits of pro-objects. For example, $[\mathrm{P}]$ contains another description of cofiltered limits. This construction is suitable for proving some of the applications below but not all of them. One disadvantage of this alternative approach is that it produces pro-objects that are very complicated in the sense that their index categories are far from being cofinite directed sets. On the other hand, the cofiltered limits constructed with the help of Theorem 3.3 are relatively small and computable.

For thoroughness, we outline here the construction of $[\mathrm{P}]$. Let $X: A \rightarrow$ pro- $\mathcal{C}: a \mapsto X^{a}$ be a diagram of pro-objects indexed by a cofiltered category $A$ such that each $X^{a}$ is indexed by a cofiltered category $I^{a}$. Roughly, the idea is that we take a diagram whose objects are of the form $X_{s}^{a}$ and whose morphisms are all possible maps $X_{s}^{a} \rightarrow X_{t}^{b}$ representing one of the pro-maps $X^{a} \rightarrow X^{b}$ in the diagram $X$. Note that even if $A$ and each $I^{a}$ are cofinite directed sets, the category $I$ is in general not a directed set.

5. Essentially levelwise maps. Our construction of limits in procategories leads to a result about the behavior of cofiltered limits on proobjects that are essentially of type $C$.

TheOREM 5.1. Let $C$ be any class of objects of a category $\mathcal{C}$, and let $c C$ be the image of this class under the constant functor $c$. The closure of $c C$ under isomorphisms and cofiltered limits is equal to the class of pro-objects in pro-e that are essentially of type $C$.

Proof. First we show that the class of pro-objects that are essentially of type $C$ is closed under cofiltered limits. Let $X$ be a cofiltered diagram of pro-objects, each of which is essentially of type $C$. We may assume that each $X^{a}$ is actually of type $C$. We use the method of [AR, Th. 1.5] to replace each $X^{a}$ by a cofinite directed pro-object that is still of type $C$. Then we use 
this same method to replace the diagram $X$ by a cofinite directed diagram with the same limit such that each $X^{a}$ is still a cofinite directed pro-object of type $C$.

Now we use the construction of Theorem 4.1. Since each $X_{s}^{a}$ belongs to $C$, each $\widetilde{X}_{s}^{a}$ belongs to $C$ (see the proof of Theorem 3.3). Since each $\widetilde{X}_{s}^{a}$ belongs to $C$, the pro-object $\widetilde{X}$ is of type $C$. Therefore, $\lim _{a}^{\text {pro }} X^{a}$ is essentially of type $C$.

Now we must show that every pro-object of type $C$ is a cofiltered limit of objects in $c C$. Let $X$ be a pro-object such that each $X_{s}$ belongs to $C$. By direct calculation of morphism sets, $X$ is isomorphic to $\lim _{s}^{\text {pro }} c X_{s}$.

Corollary 5.2. Let $C$ be any class of maps in $\mathcal{C}$. Then maps in pro- $\mathrm{C}$ essentially of type $C$ are closed under cofiltered limits.

Proof. Apply Theorem 5.1 to the category whose objects are morphisms in $\mathcal{C}$ and whose morphisms are commutative squares in $\mathcal{C}$. One must also use the fact that every pro-map has a level representation.

This corollary gives several immediate results about particular classes of maps in pro-categories that are of interest.

COROLlary 5.3. Let $\mathcal{C}$ be a "sufficiently nice" model category [EH, §2.3] or a proper model category. The class of cofibrations in the strict model structure [EH, §3.3], [I4] for pro-C is closed under cofiltered limits. The class of fibrations in the strict model structure [EH, §3.8] for ind-C is closed under filtered colimits.

COROLlary 5.4. The classes of cofibrations in the $\pi_{*}$-model structure for pro-spaces [I1], in the $\pi_{*}$-model structure for pro-spectra [I3], and in the $\pi^{*}$-model structure for pro-spectra $[\mathrm{CI}]$ are all closed under cofiltered limits. The class of fibrations in the $\pi_{*}$-model structure for ind-spectra $[\mathrm{CI}]$ is closed under filtered colimits.

The closure of cofibrations in the $\pi^{*}$-model structure for pro-spectra under cofiltered limits is a technical necessity for [CI].

Having studied cofiltered limits of essentially levelwise maps, we now proceed to retracts of such maps.

TheOREM 5.5. Let $C$ be any class of objects of a category $\mathcal{C}$. The class of objects of pro- $\mathrm{C}$ that are essentially of type $C$ is closed under retracts.

Proof. Let $Y$ be essentially of type $C$, and let $X$ be another pro-object with maps $f: X \rightarrow Y$ and $g: Y \rightarrow X$ such that the composition $g f$ is the identity. Consider the countable tower

$$
\ldots \rightarrow X \stackrel{f}{\rightarrow} Y \stackrel{g}{\rightarrow} X \stackrel{f}{\rightarrow} Y \stackrel{g}{\rightarrow} X .
$$


Since $g f$ is the identity, the limit of this tower is isomorphic to $X$. On the other hand, the limit is also isomorphic to the limit of the tower

$$
\ldots \rightarrow Y \stackrel{f g}{\longrightarrow} Y \stackrel{f g}{\longrightarrow} Y .
$$

By Theorem 5.1, this limit is essentially of type $C$.

The following corollary appears in [I1, Prop. 12.1]. The proof there is philosophically the same as the one here.

Corollary 5.6. Let $C$ be any class of maps of a category $\mathcal{C}$. The class of maps of pro- $C$ that are essentially of type $C$ is closed under retracts.

Proof. Apply Theorem 5.5 to the category whose objects are morphisms in $\mathcal{C}$ and whose morphisms are commutative squares in $\mathcal{C}$. One must also use the fact that every pro-map has a level representation.

6. Exact limits. It is a well known and useful fact that filtered colimits commute with finite limits in most familiar categories [ML, Th. IX.2.1]. Using our constructions of cofiltered limits given in Section 4, we prove that the opposite is true for pro-categories.

Theorem 6.1. Cofiltered limits commute with finite colimits in pro-e.

Proof. Let $A$ be a cofiltered index category, and let $B$ be a finite index category. Suppose given a functor

$$
X: A \times B \rightarrow \text { pro- } \mathcal{C}:(a, b) \mapsto X^{a, b} .
$$

In order to compute limits indexed by $A$, we may replace $A$ with a cofinite cofiltered index category as described at the beginning of Section 4 . In order to compute colimits indexed by $B$, we may replace $B$ by a finite loopless category; this is the usual method for rewriting any finite colimit in terms of finite coproducts and coequalizers.

Therefore, we may assume that both $A$ and $B$ are cofinite. It follows that $A \times B$ is cofinite, so we may assume that $X$ is a level representation. Thus, we have a functor

$$
X: A \times B \times I \rightarrow \mathcal{C}:(a, b, s) \mapsto X_{s}^{a, b} .
$$

Recall that finite colimits of pro-objects are computed levelwise. By Theorem 4.1 and direct computation, $\lim _{a}^{\text {pro }} \operatorname{colim}_{b}^{\text {pro }} X^{a, b}$ and $\operatorname{colim}_{b}^{\text {pro }} \lim _{a}^{\text {pro }} X^{a, b}$ are both isomorphic to the pro-object

$$
A \times I \rightarrow \mathcal{C}: s \mapsto \operatorname{colim}_{s} X_{s}^{a, b}
$$

REMARK 6.2. Pro-categories are often equivalent to the opposites of more familiar categories. For example, the category of pro-finite abelian groups is equivalent to the opposite of the category of torsion abelian groups [F, Prop. 7.5], and the category of pro-finite $k$-vector spaces is equivalent 
to the opposite of the category of $k$-vector spaces. See [CI] for an analogous stable homotopy theoretic statement. More generally, the category pro- $\mathcal{C}$ is the opposite of the pro-representable functors on $\mathcal{C}$ [AM, App. 4.2], [S]. When $\mathcal{C}$ is small (as in the case of finite abelian groups or finite $k$-vector spaces), the pro-representable functors are just the left exact functors.

If the category pro- $\mathcal{C}$ were equivalent to the opposite of a more familiar category in which filtered colimits commuted with finite limits, then Theorem 6.1 would follow. When $\mathcal{C}$ is not small, there is no clean description of the pro-representable functors. Even the categories of pro-abelian groups or pro-sets do not seem to be the opposites of any particularly familiar categories. The isomorphism types of cocompact pro-abelian groups or pro-sets do not form a set, as shown in Theorem 7.2 below. However, most familiar categories have only a set of isomorphism types of compact objects.

Let $\mathcal{A}$ be an abelian category. Then pro- $\mathcal{A}$ is again an abelian category [AM, App. 4.5], [D]. The class of monomorphisms is equal to the class of maps that have level representations by monomorphisms. The same is true for the class of epimorphisms.

Now we have a consequence of Theorem 6.1.

TheOrem 6.3. Let $\mathcal{A}$ be an abelian category. Then cofiltered limits are exact in the category pro- $\mathcal{A}$.

Proof. Limits are always left exact. Cofiltered limits are right exact by Theorem 6.1.

The surprising part of the previous theorem is that filtered limits are right exact. This is contrary to what occurs in most familiar abelian categories. The failure of epimorphisms to be closed under cofiltered limits is the source of the higher $\lim ^{i}$ functors.

Filtered colimits in the category pro- $\mathcal{A}$ need not be exact. This is another notable difference between pro-categories and most familiar abelian categories. It may be possible to define higher derived colimit functors colim $^{i}$ in this context. However, pro- $\mathcal{A}$ does not have enough projectives $[\mathrm{Z}]$, so the usual approach to derived functors does not work.

EXAMPLE 6.4. We give an example of the inexactness of filtered colimits in the category of pro-abelian groups. Let $A$ be the free abelian group generated by the countable basis $\left\{a_{0}, a_{1}, \ldots\right\}$. For $n \geq m \geq 0$, let $A[m, n]$ be the subgroup of $A$ generated by $\left\{a_{m}, a_{m+1}, \ldots, a_{n}\right\}$. For $m \geq 0$, let $A[m, \infty)$ be the subgroup of $A$ generated by $\left\{a_{m}, a_{m+1}, \ldots\right\}$.

Let $X$ be the pro-abelian group

$$
\ldots \rightarrow A[0,2] \rightarrow A[0,1] \rightarrow A[0,0] .
$$

The structure maps of $X$ are the obvious projections. 
Recall the constant functor $c: \mathcal{C} \rightarrow$ pro- $\mathcal{C}$. For $n \geq 0$, there is a map $f_{n}: c A[0, n] \rightarrow X$ of pro-abelian groups. This map is the element of

$$
\begin{aligned}
\lim _{m} \operatorname{Hom}(A[0, n], A[0, m]) & \cong \operatorname{Hom}\left(A[0, n], \lim _{m} A[0, m]\right) \\
& \cong \operatorname{Hom}\left(A[0, n], \prod_{m} \mathbb{Z} a_{m}\right)
\end{aligned}
$$

given by the obvious inclusion $A[0, n] \rightarrow \prod_{m} \mathbb{Z} a_{m}$. Each map $f_{n}$ is a monomorphism in the category of pro-abelian groups because it has a level representation

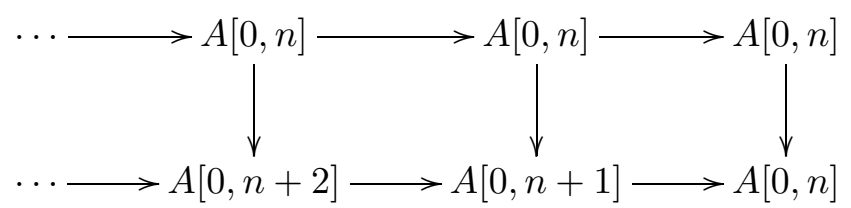

in which each vertical map is a monomorphism.

Note that the maps $f_{n}$ are compatible with the inclusions $c A[0, n] \rightarrow$ $c A[0, n+1]$ in the sense that the diagram

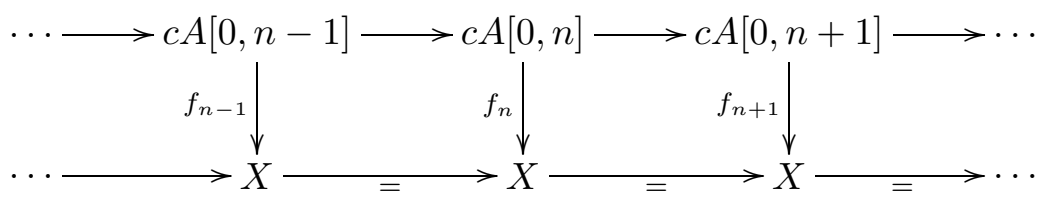

of pro-abelian groups commutes. The colimit of the first row is $c A$ because $A$ is the union of the groups $A[0, n]$ and because $c$ commutes with colimits since it is a left adjoint. The colimit of the second row is just $X$. Thus, the colimit of the above diagram is a map $f: c A \rightarrow X$. Now $f$ is a filtered colimit of the monomorphisms $f_{n}$, but we claim that $f$ is not a monomorphism.

In order to show that $f$ is not a monomorphism, we construct a nonzero map $g: Y \rightarrow c A$ such that the composition $f g$ is zero. Let $Y$ be the pro-abelian group

$$
\ldots \rightarrow A[2, \infty) \rightarrow A[1, \infty) \rightarrow A[0, \infty) .
$$

The structure maps of $Y$ are the obvious inclusions. Let $g$ be the element of

$$
\underset{n}{\operatorname{colim}} \operatorname{Hom}(A[n, \infty), A)
$$

represented by any of the obvious inclusions $A[n, \infty) \rightarrow A$. Then $g$ is not the zero map because each map $A[n, \infty) \rightarrow A$ is non-zero. On the other hand, the composition $Y \rightarrow X$ is the zero element of

$$
\lim _{m} \operatorname{colim}_{n} \operatorname{Hom}(A[n, \infty), A[0, m])
$$

because for every $m$ and every $n>m$, the composition $A[n, \infty) \rightarrow A \rightarrow$ $A[0, m]$ is zero. 
7. Cocompact objects. As another application of our explicit computation of cofiltered limits, we describe completely in this section the cocompact objects of pro-e.

Recall that an object $X$ of a category is compact if for every filtered system $Y$, the map

$$
\underset{a}{\operatorname{colim}} \operatorname{Hom}\left(X, Y^{a}\right) \rightarrow \operatorname{Hom}\left(X, \underset{a}{\operatorname{colim}} Y^{a}\right)
$$

is an isomorphism. We dualize this notion as follows.

Definition 7.1. An object $X$ of a category is cocompact if for every cofiltered system $Y$, the map

$$
\underset{a}{\operatorname{colim}} \operatorname{Hom}\left(Y^{a}, X\right) \rightarrow \operatorname{Hom}\left(\lim _{a} Y^{a}, X\right)
$$

is an isomorphism.

At first glance, this appears to be a strange definition. Note that $X$ is cocompact if and only if $X$ is compact in the opposite category.

THEOREM 7.2. A pro-object is cocompact if and only if it is isomorphic to a constant pro-object.

Proof. First consider a constant pro-object $c X$. Let $Y$ be an arbitrary cofiltered system of pro-objects indexed by $A$. As described at the beginning of Section 4, we may assume that $A$ is cofinite. Thus, we may take $Y$ to be a level representation. It follows by Theorem 4.1 and direct calculation that both $\operatorname{Hom}_{\text {pro }}\left(\lim _{a}^{\text {pro }} Y^{a}, c X\right)$ and $\operatorname{colim}_{a} \operatorname{Hom}_{\text {pro }}\left(Y^{a}, c X\right)$ are equal to $\operatorname{colim}_{a} \operatorname{colim}_{s} \operatorname{Hom}\left(Y_{s}^{a}, X\right)$. This shows that $c X$ is cocompact.

Now suppose that $X$ is a cocompact pro-object. Note that $X$ is isomorphic to $\lim _{s}^{\text {pro }} c X_{s}$, so the map

$$
\phi: \operatorname{colim}_{a} \operatorname{Hom}_{\text {pro }}\left(c X_{s}, X\right) \rightarrow \operatorname{Hom}_{\text {pro }}(X, X)
$$

is an isomorphism. Let $f: c X_{s} \rightarrow X$ be any map such that $\phi(f)$ is equal to the identity on $X$, and let $g: X \rightarrow c X_{s}$ be the natural map.

By definition of $\phi$, the composition $f g$ is the identity on $X$. This shows that $X$ is a retract of the constant pro-object $c X_{s}$. Applying the following proposition, we conclude that $X$ is in fact isomorphic to a constant proobject.

The next proposition is used in the proof of Theorem 7.2, but it is interesting for its own sake.

Proposition 7.3. A retract of a constant pro-object is isomorphic to a constant pro-object.

Proof. Let $X$ be a pro-object that is a retract of a constant pro-object $c Y$. This means that we have maps $f: X \rightarrow c Y$ and $g: c Y \rightarrow X$ such that the composition $g f$ is the identity on $X$. 
The map $g$ factors into the composition of a map $g^{\prime}: c Y \rightarrow c \lim _{s}^{\mathrm{e}} X_{s}$ followed by the canonical map $\varepsilon: c \lim _{s}^{\mathrm{e}} X_{s} \rightarrow X$. In fact $\varepsilon$ is an isomorphism with inverse $g^{\prime} f$. The composition $\varepsilon\left(g^{\prime} f\right)$ is the identity because $g f$ is the identity. For the composition $\left(g^{\prime} f\right) \varepsilon$, one can show that the map $\lim ^{\mathrm{e}}\left(g^{\prime} f \varepsilon\right)$ is the identity on $\lim _{s}^{\mathrm{e}} X_{s}$. This shows that $\left(g^{\prime} f\right) \varepsilon$ is also the identity.

8. Colimits in pro-categories. We assume in this section that $\mathcal{C}$ is cocomplete. The category pro- $\mathrm{C}$ is also cocomplete [I1, Prop. 11.1], but colimits in pro- $\mathcal{C}$ are difficult to compute in general. See [B] for a special situation. We study a different special situation in which explicit computations are possible. In particular, we compute colimits in pro-e indexed by cofinite categories. At first glance, this does not seem to be especially useful, so we provide two examples.

EXAMPLE 8.1. Consider the diagram for calculating the realization of a simplicial space $X$. This diagram has one object $X_{n} \otimes \Delta[n]$ for each $n \geq 0$ and one object $X_{n} \otimes \Delta[m]$ for each simplicial operator $\phi:[m] \rightarrow[n]$. The maps of the diagram are of two types. The first type is of the form $\operatorname{id} \otimes \phi_{*}: X_{n} \otimes \Delta[m] \rightarrow X_{n} \otimes \Delta[n]$, and the second type is of the form $\phi^{*} \otimes$ id : $X_{n} \otimes \Delta[m] \rightarrow X_{m} \otimes \Delta[m]$.

The colimit of this diagram is the realization $|X|$ of $X$. Note that the diagram is cofinite because each object $X_{n} \otimes \Delta[n]$ is the source of zero non-identity maps and each object $X_{n} \otimes \Delta[m]$ is the source of two nonidentity maps. Therefore, the techniques of this section apply to calculating realizations of simplicial pro-objects.

ExAmPle 8.2. Consider a countable sequence

$$
X_{0} \stackrel{f_{0}}{\longrightarrow} X_{1} \stackrel{f_{1}}{\longrightarrow} X_{2} \stackrel{f_{2}}{\longrightarrow} \ldots
$$

In order to calculate colim ${ }^{\text {pro }} X$, we may take the colimit of the diagram

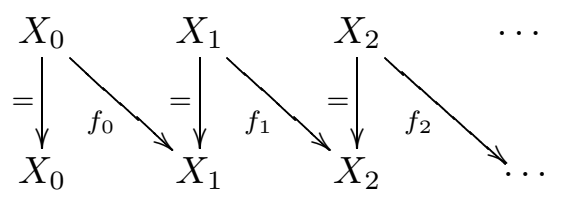

which is cofinite. Thus, the techniques of this section apply to calculating colimits of countable sequences.

Let $A$ be a cofinite category, and let $I$ be a cofinite directed set. Recall that an arbitrary product of cofiltered categories is again cofiltered. Similarly, an arbitrary product of directed sets is again a directed set, but infinite products do not preserve cofiniteness. Consider the subset $K$ of $\prod_{A} I$ consisting of tuples $\left(s_{a}\right)$ such that $s_{a} \geq s_{b}$ when $a \geq b$. Define a partial ordering 
on $K$ by $\left(s_{a}\right) \geq\left(t_{a}\right)$ if $s_{a} \geq t_{a}$ for all $a$. This is the ordering that $K$ inherits as a subset of $\prod_{A} I$.

LEMMA 8.3. The set $K$ is directed.

Proof. Let $\left(s_{a}\right)$ and $\left(t_{a}\right)$ be any two elements of $K$. We construct a common refinement $\left(u_{a}\right)$ by induction on $a$. This is possible since $A$ is cofinite. Suppose that $u_{b}$ has already been determined for $b<a$ such that $u_{b} \geq s_{b}$ and $u_{b} \geq t_{b}$. Choose $u_{a}$ such that $u_{a} \geq s_{a}, u_{a} \geq t_{a}$, and $u_{a} \geq u_{b}$ for all $b<a$. This is possible because there are only finitely many conditions on $u_{a}$.

Note that $K$ is not necessarily cofinite.

LEMma 8.4. The inclusion $K \rightarrow \prod_{A} I$ is cofinal.

Proof. Given an element $\left(s_{a}\right)$ of $\prod_{A} I$, we must find an element $\left(t_{a}\right)$ of $K$ such that $\left(t_{a}\right) \geq\left(s_{a}\right)$. Suppose that $t_{b}$ has already been chosen for $b<a$ such that $t_{b} \geq s_{b}$. Choose $t_{a}$ such that $t_{a} \geq s_{a}$ and $t_{a} \geq t_{b}$ for all $b<a$. This is possible because there are only finitely many conditions on $t_{a}$.

LEMma 8.5. The forgetful functor $U^{a}: K \rightarrow I:\left(s_{a}\right) \mapsto s_{a}$ is cofinal.

Proof. The projection functor $\prod_{A} I \rightarrow I$ is cofinal. By Lemma 8.4, the functor $U^{a}$ is a composition of two cofinal functors, so it is also cofinal.

Let $X: A \rightarrow$ pro- $\mathrm{C}$ be a functor. By Theorem 3.3, we know that $X$ has a level representation $\widetilde{X}: A \times I \rightarrow \mathcal{C}$ for some cofinite directed set $I$. We now define a functor $\bar{X}: A \times K \rightarrow \mathcal{C}$. Let $s=\left(s_{a}\right)$ be an element of $K$. For every object $a$ in $A$, define $\bar{X}_{s}^{a}$ to be $\widetilde{X}_{s_{a}}^{a}$. For every map $a \rightarrow b$ in $A$, define $\bar{X}_{s}^{a} \rightarrow \bar{X}_{s}^{b}$ to be either composition in the commuting square

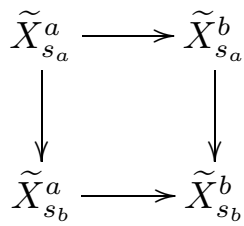

Note that the vertical maps are the structure maps of the pro-objects $\widetilde{X}^{a}$ and $\widetilde{X}^{b}$. Here we use the fact that $s_{a} \geq s_{b}$ since $s$ belongs to $K$. Also note that the horizontal maps come from the level representation $\widetilde{X}^{a} \rightarrow \widetilde{X}^{b}$ of the map $X^{a} \rightarrow X^{b}$.

One can verify that $\bar{X}$ is indeed a functor by a straightforward diagram chase.

THEOREM 8.6. The pro-object $Z$ indexed by $K$ given by $Z_{s}=\operatorname{colim}_{a}^{\mathrm{e}} \bar{X}_{s}^{a}$ is the colimit in the category pro- $\mathrm{C}$ of the functor $X$. 
Proof. Let $W$ be an arbitrary pro-object. Then direct computation with the definition of morphism sets in pro- $\mathcal{C}$ shows that

$$
\operatorname{Hom}_{\text {pro }}(Z, W) \cong \lim _{a \in A} \operatorname{Hom}_{\text {pro }}\left(X^{a}, W\right) .
$$

This uses Lemma 8.5 and the surprising fact that the canonical map

$$
\operatorname{colim}_{s \in K} \lim _{a \in A} \operatorname{Hom}_{\mathcal{C}}\left(\widetilde{X}_{s_{a}}^{a}, W_{t}\right) \rightarrow \lim _{a \in A} \operatorname{colim}_{s \in K} \operatorname{Hom}_{\mathcal{C}}\left(\widetilde{X}_{s_{a}}^{a}, W_{t}\right)
$$

is an isomorphism, which is justified by Lemma 8.4.

\section{References}

[AR] J. Adámek and J. Rosický, Locally Presentable and Accessible Categories, London Math. Soc. Lecture Note Ser. 189, Cambridge Univ. Press, 1994.

[AHJM] J. F. Adams, J.-P. Haeberly, S. Jackowski and J. P. May, A generalization of the Segal conjecture, Topology 27 (1988), 7-21.

[SGA4] M. Artin, A. Grothendieck et J.-L. Verdier, Théorie des topos et cohomologie étale des schémas (SGA 4), tome 1, Lecture Notes in Math. 269, Springer, 1972.

[AM] M. Artin and B. Mazur, Étale Homotopy, Lecture Notes in Math. 100, Springer, 1969 .

[B] D. Blanc, Colimits for the pro-category of towers of simplicial sets, Cahiers Topologie Géom. Différentielle Catég. 37 (1996), 258-278.

[CI] J. D. Christensen and D. C. Isaksen, Duality and pro-spectra, in preparation.

[CJS] R. L. Cohen, J. D. S. Jones and G. B. Segal, Floer's infinite-dimensional Morse theory and homotopy theory, in: The Floer Memorial Volume, Progr. Math. 133, Birkhäuser, 1995, 297-325.

[CP] J.-M. Cordier and T. Porter, Shape Theory: Categorical Methods of Approximation, Ellis Horwood Ser. Math. Appl., Ellis Horwood, 1989.

[D] J. Duskin, Pro-objects (after J.-L. Verdier), in: Dualité de Poincaré (Séminaire Heidelberg-Strasbourg, 1966/67), Publ. I.R.M.A. Strasbourg 3, Univ. de Strasburg, 1969, Exposé 6 .

[EH] D. A. Edwards and H. M. Hastings, Čech and Steenrod Homotopy Theories with Applications to Geometric Topology, Lecture Notes in Math. 542, Springer, 1976.

[F] E. M. Friedlander, Étale homotopy of simplicial schemes, Ann. of Math. Stud. 104, Princeton Univ. Press, 1982.

[I1] D. C. Isaksen, A model structure for the category of pro-simplicial sets, Trans. Amer. Math. Soc. 353 (2001), 2805-2841.

[I2] - Etale realization on the $\mathbb{A}^{1}$-homotopy theory of schemes, submitted.

[I3] - The pro-Atiyah-Hirzebruch spectral sequence, in preparation.

[I4] - Strict model structures for pro-categories, in: Proc. Internat. Conf. in Algebraic Topology (Skye, 2001), to appear.

[ML] S. Mac Lane, Categories for the Working Mathematician, Grad. Texts in Math. 5, Springer, 1971.

[MS] S. Mardešić and J. Segal, Shape Theory: The Inverse System Approach, NorthHolland Math. Library 26, North-Holland, 1982. 
[Me] C. V. Meyer, Approximation filtrante de diagrammes finis par Pro-C, Ann. Sci. Math. Québec 4 (1980), 35-57.

[Mo] F. Morel, Ensembles profinis simpliciaux et interprétation géométrique du foncteur T, Bull. Soc. Math. France 124 (1996), 347-373.

[P] T. Porter, Essential properties of pro-objects in Grothendieck categories, Cahiers Topologie Géom. Différentielle 20 (1979), 3-57.

[S] H. B. Stauffer, A relationship between left exact and representable functors, Canad. J. Math. 23 (1971), 374-380.

[V] J.-L. Verdier, Équivalence essentielle des systèmes projectifs, C. R. Acad. Sci. Paris 261 (1965), 4950-4953.

[Z] S. Zdravkovska, On injective and projective objects in pro-abelian categories, Glas. Mat. Ser. III 14 (34) (1979), 223-226.

Department of Mathematics

University of Notre Dame

Notre Dame, IN 46556, U.S.A.

E-mail: isaksen.1@nd.edu

Received 30 April 2002 